Z Herz- Thorax- Gefäßchir 2020 · 34:219-220 https://doi.org/10.1007/s00398-020-00382-2 Online publiziert: 23. Juli 2020

(c) Springer Medizin Verlag GmbH, ein Teil von Springer Nature 2020

\author{
A. Welz \\ Halblech, Deutschland
}

\title{
Fortschritt in der Herzchirurgie im Wandel der Zeit
}

Liebe Leserinnen und Leser,

die vorliegende Ausgabe 4/2020 der Zeitschrift für Herz-, Thorax- und Gefäßchirurgie überspannt mit den Themen ihrer Artikel annähernd ein Jahrhundert. Dies trifft zweifelsfrei zu, wenn man den Beginn der Herzchirurgie auf das Jahr 1938 datiert. In diesem Jahr führte der Kinderchirurg Robert E. Gross am Boston Children's Hospital den ersten Verschluss eines Ductus arteriosus persistens durch und eröffnete somit die Möglichkeit der Behandlung einer Herzerkrankung, die neben der zwangsläufigen Entstehung einer pulmonalen Hypertonie und Rechtsherzinsuffizienz in der damaligen Zeit durch eine nicht unbeträchtliche Rate an Endokarditis kompliziert war.

Stellt man den historischen Artikel von Kollegen Ulmer dem Beitrag zu Robotertechniken in der Herzchirurgie von M. Misfeld und T.D. Yan gegenüber, wird der Wandel der angestrebten Ziele des Fortschritts in der Herzchirurgie unmittelbar deutlich. In der Anfangszeit der Herzchirurgie stand die Ausweitung der Behandlungsmöglichkeiten auf immer mehr Erkrankungen des Herzens im Vordergrund. Ziel war, bislang unbehandelbare Patienten mit lebensqualitätund lebenszeitlimitierenden Erkrankungen therapeutische Optionen anbieten zu können. H.E. Ulmer schildert eindrucksvoll den beruflichen Werdegang von Robert E. Gross, der sich während seiner gesamten chirurgischen Karriere der Behandlung des ductus arteriosus Botalli widmete. Dies reichte von der Beobachtung schwerer Verläufe dieser bislang unbehandelbaren Erkrankung währen einer Assistenzzeit in der Pathologie über die grundsätzliche Idee eines möglichen Verschlusses sowie sorg- fältigen experimentellen Vorversuchen bis zur ersten erfolgreichen klinischen Umsetzung im Operationssaal, erwähnenswert trotz Verbot durch seinen chirurgischen Lehrer. Es wurden dann in rascher Folge weitere Behandlungsmöglichkeiten eröffnet. Erwähnt sei hier nur die Einführung der Palliativbehandlung der Fallot-Tetralogie durch Schaffen einer Verbindung zwischen systemarteriellem und pulmonalem Gefäßbett durch Alfred Blalock im Jahr 1944, letztlich eine Umkehrung der Gross-Operation. Der Eingriff wurde von der Kinderärztin Helen Taussig vorgeschlagen, die in ihrer Praxis wiederum beobachtet hatte, dass Kinder mit Fallot-Tetralogie und zugleich offenem Ductus Botalli weniger unter zyanotischen Anfällen litten

In heutiger Zeit ist die weitaus überwiegende Mehrzahl der Herz- und Gefäßerkrankungen einer chirurgischen Therapie zugänglich. Medizinischer Fortschritt bezieht sich nun v. a. auf die Verfeinerung operativer Techniken mit dem Ziel der Verringerung von Morbidität und Mortalität der durchgeführten Operationen. Der Artikel „Robotertechniken in der Herzchirurgie" beschreibt die Möglichkeit der Anwendung dieser Verfahren in unserem Fachgebiet. Dabei werden neben der technischen Beschreibung und ausgewählten Indikationen Vor- und Nachteile kritisch gegenübergestellt. Das Verfahren muss letztlich im Sinne eines "dexterity enhancement" gesehen werden. Vergleichbar der Lupenbrille, die die Leistungsfähigkeit des menschlichen Auges verbessert, erhöht das Robotersystem die Geschicklichkeit der menschlichen Hand und ermöglicht somit präzises Operieren auf engstem Raum. Allerdings wird dieser Vorteil z.T. durch das fehlende taktile Feedback 
wieder aufgehoben. Darüber hinaus erfordert die Anwendung von Robotersystemen extensives spezialisiertes Training. Die Indikation ist auch nach Aussage der Autoren auf streng selektionierte Fälle beschränkt. Der valide wissenschaftliche Beweis einer Ergebnisverbesserung wurde bislang nicht erbracht. All dies dürfte letztlich der Grund sein, dass sich die Technik wie im Artikel deutlich beschrieben noch nicht weit verbreitet in der Herzchirurgie durchsetzen konnte.

Zusammengefasst, in der Anfangszeit der Herzchirurgie stand dem akademischen Chirurgen ein weites Feld an zu entwickelnden Behandlungsmöglichkeiten offen, der erste Einsatz erforderte aber neben sorgfältiger Vorarbeit erheblichen Mut des Operateurs aber v.a. auch der betroffenen Patientinnen/Patienten bzw. deren Eltern. Demgegenüber erscheinen die Möglichkeiten der Generierung weiterer Fortschritte in der Chirurgie heute kleinteiliger, v. a. bezogen auf moderne technische Entwicklungen in den Gebieten EDV-gestützter Verfahren zu Bildgebung, Navigation und OPTechnik. Zugleich steigt bei allen diesen technischen Neuerungen die Bedeutung ökonomischer Kosten- Nutzen Abschätzungen.

Neben den von mir in diesem Editorial hervorgehobenen Artikeln enthält diese Ausgabe der $Z f H T G$ wie gewohnt weitere spannende Rubrikenbeiträge. Die Autoren mögen mir verzeihen, diese nicht in meine Betrachtungen mit einbezogen $\mathrm{zu}$ haben.

Sehr geehrte, liebe Leserinnen und Leser, ich wünsche Ihnen viel Freude und Erkenntnisgewinn bei Lektüre und Studium der Ausgabe 4/2020 der Zeitschrift für Herz-, Thorax- und Gefäßchirurgie.

Ihr<smiles>CCCCCCCCCCCCc1ccccc1C</smiles>

Prof. Dr. Armin Welz

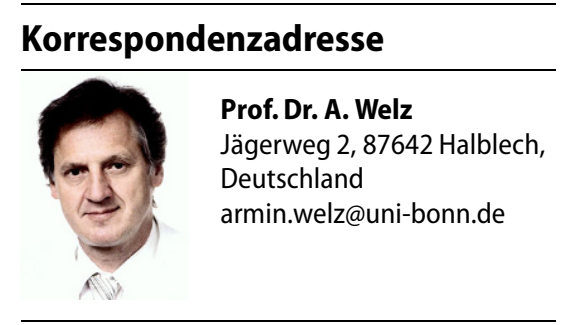

Interessenkonflikt. A. Welz gibt an, dass kein Interessenkonflikt besteht.

\section{Aktuelle Informationen}

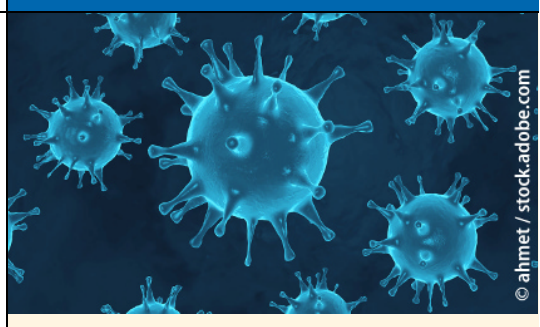

\section{Aktuelle Entwicklung zu COVID-19 bei Springer Nature und Springer Medizin}

Springer Nature und Springer Medizin unterstützen die globale Reaktion auf die COVID-19-Pandemie, indem ein schneller und direkter $\mathrm{Zu}$ gang zu den neuesten verfügbaren Forschungsergebnissen und Daten ermöglicht wird.

Auf der Homepage SpringerMedizin.de finden Sie ein immer aktuelles Dossier mit Beiträgen, Forschungsarbeiten und Ergebnissen zu SARS-CoV-2 sowie relevanten Links.

Darin z.B. auch die kürzlich publizierte

Empfehlung von DIVI, DGIIN, DGAI und DGP zur Intensivtherapie von Patienten mit COVID-19.

Springer Nature arbeitet mit globalen Organisationen zusammen, und verlinkt über SpringerNature.com/de auf eine eigene Landingpage mit einer Vielzahl an Information sowie freiem Zugriff auf die COVID-19-Contentplattformen von Nature Research, BioMed Central (BMC) und Springer.

Das Dossier zu Coronavirus / Covid-19 von Springer Medizin finden Sie hier: www.springermedizin.de/covid-19

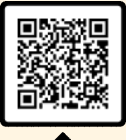

